\title{
KINEMATICS OF THE ION TAIL OF COMET P/SWIFT-TUTTLE
}

\author{
Hyron SPINRAD, Michael E. Brown, AND Christopher M. JohnS \\ Department of Astronomy, University of California, Berkeley, California 94720 \\ Electronic mail: spinrad@bigz.berkeley.edu, brown@bigz.berkeley.edu, cjohns@astro.berkeley.edu \\ Received 1994 March 24; revised 1994 May 17
}

\begin{abstract}
We have obtained long-slit high resolution spectra of the $\mathrm{H}_{2} \mathrm{O}^{+} 6199 \AA$ complex in the near tail of comet $\mathrm{P} /$ Swift-Tuttle. The observations were made using the Hamilton echelle spectrometer fed by the Lick Observatory $0.6 \mathrm{~m}$ coudé auxiliary telescope. For most of our observations, the spectral slit was aligned along the Sun-tail axis and the cometary nucleus was placed at one end of the slit, giving us spectra having the spatial and spectral resolution needed to measure the radial velocity and velocity dispersion continuously down the cometary tail out to a distance of $4 \times 10^{5} \mathrm{~km}$. The radial velocities confirm the earlier more restricted observations by Rauer \& Jockers [Icarus, 102, 117 (1993)] and by Wyckoff \& Lindholm (1994) showing that the tail motions are indeed bulk flows in the antisolar direction. Out to $3 \times 10^{5} \mathrm{~km}$ in the tail typical bulk flows are at a speed of $\sim 30 \mathrm{~km} \mathrm{~s}^{-1}$. The velocity dispersion, $\left(\sigma_{r}\right)$, of the $\mathrm{H}_{2} \mathrm{O}^{+}$lines follows a pattern that is quite systematic; $\sigma_{r}$ is smallest near the cometary nucleus, and steadily increases down the tail. The highest velocity dispersions are found ahead of the nucleus and off the tail axis. These velocity dispersions are equivalent to ion temperatures ranging from $10^{5}$ to $10^{6} \mathrm{~K}$. We note a clear anticorrelation between the $\mathrm{H}_{2} \mathrm{O}^{+}$line intensities (related to the ion density) and the bulk flow and dispersion velocities; direct mass loading of the solar wind by the observed water ions may be responsible. We discuss several approximate equipartition methods used to infer local magnetic fields induced by the interaction of the cometary ions with the solar wind particle/field stream. Typical fields derived are near 50 $\mathrm{nT}$. The measured tailward accelerations are consistent with this order of magnitude $B$ field.
\end{abstract}

\section{INTRODUCTION AND OBSERVATIONS}

While cometary ion tails make attractive photographs, the physical study of the ions and of their kinematics has been restricted. The reasons for this lack of data are not surprising; spacecraft visit comets only rarely and for very brief intervals in their flythroughs. Ground-based spectroscopy and imaging of the faint cometary ionic emission has only recently approached a standard where plasma kinematics could be discerned. Early work by Herbig (1990) showed that velocities in Comet Halley were small near the nucleus. Thus, until the research of Rauer \& Jockers (1993) and Wyckoff \& Lindholm (1994), little proof was available to confirm that the proper motions of tail condensations were really bulk flows down the tail as opposed to a wave phenomenon. Of course, much of the early theory makes interesting historical reading: a prescient paper is by Harwit \& Hoyle (1962).

In this paper we present high-resolution long-slit spectra from which we measure radial velocities and velocity dispersions, leading us to checks on the extant kinematic models for the relatively abundant water ion molecules in the sunside coma and into the cometary tail of comet $\mathrm{P} / \mathrm{Swift}-$ Tuttle. Though we have no images of the comet from the precise time of the observations, a series of amateur images (shown in Sky and Telescope, January and March, 1993) shows a relatively undisturbed tail in the weeks surrounding the observations.

In order to obtain the desired high spectral resolution and large scale on the plane of the sky we required a slightly unusual spectrographic/telescopic format for our observations of Comet P/Swift-Tuttle in late 1992. We employed the Lick Observatory $0.6 \mathrm{~m}$ coudé auxiliary telescope (CAT) coupled to the Hamilton echelle spectrograph (Vogt 1987) used in a single order, long-slit mode (isolating order $n=92$ with a special narrow-band filter centered at $\lambda 6199 \AA$ ). The spectra were recorded on a Ford $2048 \times 2048$ CCD chip that was binned by a factor of 4 in the spatial dimension and a factor of 2 in the wavelength dimension to decrease the readout noise of the chip with respect to the total signal from the comet and the night sky. The nucleus of comet P/SwiftTuttle was usually placed near one end of the long slit, and the slit position angle was aligned along the tailward projection of the Sun-comet vector. On two occasions we obtained useful spectra with the slit perpendicular to the radius vector, some $2^{\prime}$ down the tail.

The spectrograms achieved a spectral resolution of $\lambda / \Delta \lambda$ $($ FWHM) $\simeq 33000$ (corresponding to a velocity resolution of $9 \mathrm{~km} \mathrm{~s}^{-1}$ at FWHM or $4.3 \mathrm{~km} \mathrm{~s}^{-1}$ for the $1 \sigma$ Gaussian width, $\sigma_{0}$ ). The binned spatial resolution was about $10 \mathrm{arcsec} /$ pixel. That angle projected to $10^{4} \mathrm{~km}$ at the comet's typical geocentric distance in 1992 November and early December. The total available unvignetted slit length was 6 arcmin (roughly $4 \times 10^{5} \mathrm{~km}$ in projection at $\mathrm{P} / \mathrm{Swift}-\mathrm{Tuttle}$ ), and the spectrograph slit width was 7.5 in angle. The observational parameters of the spectra are listed in Table 1. Preliminary results from these observations are discussed in Brown et al. (1993).

The raw two-dimensional spectra were reduced identically: first, the bias and dark currents were subtracted. Then the spectral images were flatfielded using the spectrum of a quartz lamp to map the pixel-to-pixel sensitivity variations of the CCD. Larger-scale nonuniformities in the transmission 
TABLE 1. Journal of Swift-Tuttle observations.

\begin{tabular}{|c|c|c|c|c|c|c|c|c|}
\hline $\begin{array}{l}\text { UT Date and Time } \\
\text { (Center of Exp.) }\end{array}$ & $\begin{array}{l}\text { Exposure } \\
\text { (seconds) }\end{array}$ & $\begin{array}{l}r^{(1)} \\
(\mathrm{AU})\end{array}$ & $\begin{array}{c}\dot{r}^{(2)} \\
\left(\mathrm{km} \mathrm{s}^{-1}\right)\end{array}$ & $\beta^{(3)}$ & $\begin{array}{l}\Delta^{(4)} \\
(\mathrm{AU})\end{array}$ & $\begin{array}{c}\dot{\Delta}^{(5)} \\
\left(\mathrm{km} \mathrm{s}^{-1}\right)\end{array}$ & $\begin{array}{l}\text { Filter } \\
(\AA)\end{array}$ & Slit $^{(6)}$ \\
\hline 1992 Nov. $23,2: 57$ & 3600 & 1.013 & -9.58 & 49.8 & 1.268 & 21.3 & 6199 & 1 \\
\hline 1992 Nov. $26,3: 06$ & 3600 & 0.998 & -8.25 & 48.4 & 1.307 & 24.0 & 6199 & 1 \\
\hline 1992 Nov. $28,2: 22$ & 3600 & 0.989 & -7.34 & 47.4 & 1.335 & 25.5 & 6199 & 1 \\
\hline 1992 Nov. $28,3: 26$ & 3000 & 0.989 & -7.32 & 47.4 & 1.336 & 25.5 & 6310 & 1 \\
\hline 1992 Nov. $29,2: 05$ & 3150 & 0.985 & -6.86 & 46.7 & 1.350 & 26.2 & 6199 & 1 \\
\hline 1992 Nov. $30,2: 41$ & 3600 & 0.981 & -6.36 & 46.2 & 1.366 & 26.8 & 6199 & 1 \\
\hline 1992 Nov. $30,3: 35$ & 3800 & 0.981 & -6.34 & 46.1 & 1.367 & 26.8 & 5190 & 1 \\
\hline 1992 Dec. 01, 2:56 & 3600 & 0.977 & -5.89 & 45.5 & 1.382 & 27.4 & 6199 & 1 \\
\hline 1992 Dec. 01, 3:43 & 2900 & 0.977 & -5.85 & 45.5 & 1.383 & 27.4 & 6563 & 1 \\
\hline 1992 Dec. 02, 2:34 & 3000 & 0.974 & -5.37 & 44.8 & 1.398 & 27.9 & 6199 & 2 \\
\hline 1992 Dec. 02, 3:32 & 3000 & 0.974 & -5.35 & 44.8 & 1.398 & 27.9 & 6199 & 2 \\
\hline 1992 Dec. $19,2: 29$ & 3200 & 0.965 & +3.59 & 30.4 & 1.687 & 29.2 & 6199 & 2 \\
\hline 1992 Dec. $22,2: 22$ & 2681 & 0.973 & +5.14 & 27.6 & 1.736 & 28.3 & 6199 & 2 \\
\hline 1992 Dec. $23,2: 21$ & 1831 & 0.976 & +5.64 & 26.7 & 1.753 & 28.0 & 6199 & 1 \\
\hline 1992 Dec. $24,2: 18$ & 2030 & 0.979 & +6.13 & 25.6 & 1.768 & 27.7 & 6199 & 1 \\
\hline
\end{tabular}

Notes: (1) $r$ is the comet-sun distance; (2) $\dot{r}$ is the component of velocity along the sun-comet line; (3) $\beta$ is the sun-comet-earth angle; (4) $\Delta$ is the earth-comet distance; (5) $\dot{\Delta}$ is the velocity along the earth-comet vector; (6) a value of 1 indicates the slit was aligned along the tail of the comet and a value of 2 indicates that the slit was aligned perpendicular to the tail $2^{\prime}$ behind the nucleus, on the tail-side.

along the spatial dimension of the slit were removed by dividing each cometary spectrogram by the spatial distribution measured with the continuum of a day-sky spectrum. The wavelength scales and reference line profiles for our spectra were calibrated from spectra of a thorium-argon lamp; the Hamilton spectrograph provides a very stable velocity system, and the absolute precision of our radial velocities are near $\pm 1 \mathrm{~km} \mathrm{~s}^{-1}$.

Figure 1 illustrates the two-dimensional spectrum around

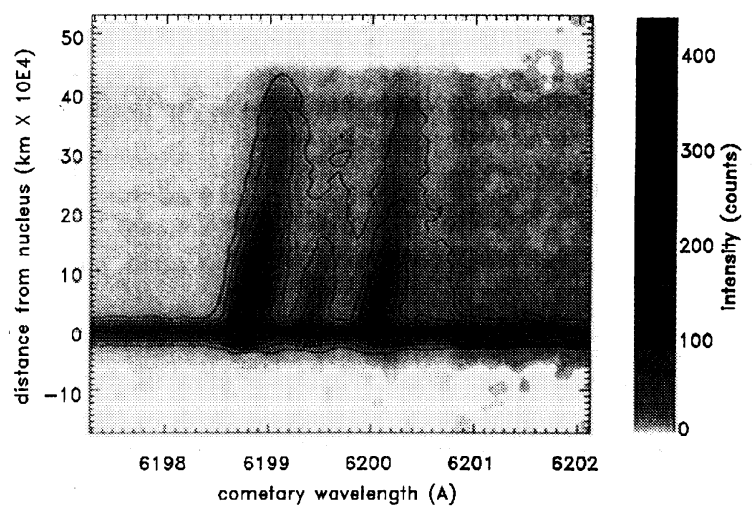

Fig. 1. A long slit $\mathrm{H}_{2} \mathrm{O}^{+}$spectrum of Comet $\mathrm{P} / \mathrm{Swift}-\mathrm{Tuttle}$ from the night of 1992 November 26 . The acceleration of the plasma tail is clearly visible in this spectrum as the shift to longer wavelengths of the $\mathrm{H}_{2} \mathrm{O}^{+}$emission down the tail. The spectral slit was aligned along the Sun-comet vector, so the spatial dimension of the spectrum is proportional to distance from the nucleus along this vector. The continuous emission at the position of the nucleus is solar continuum light scattered from cometary dust particles. At this wavelength, a Doppler shift of $1 \AA$ corresponds to a velocity of 48 $\mathrm{km} \mathrm{s}^{-1}$. the strongest $\mathrm{H}_{2} \mathrm{O}^{+}(8-0,6199 \AA$ band) lines for Comet $\mathrm{P} /$ Swift-Tuttle from the night of 30 November 1992 (UT). The emission from the water ion lines is clearly visible as the four strong and slanted emission lines; the primary goal of this paper is to measure and interpret the Doppler slant, the linewidths, and the intensities of the $\mathrm{H}_{2} \mathrm{O}^{+}$lines. A line identification list for the four emission lines is given in Table 2 . Figure 2 compares the one-dimensional spectra extracted at three points along the cometary tail and shows the excellent signal to noise achieved in these observations, which were usually integrations of $\sim 3000 \mathrm{~s}$.

To obtain precise velocity measurements we used a crosscorrelation method where the spectrum at each spatial location is cross correlated with the spectrum at each other location to obtain a velocity shift. From this system of mutual cross correlations we obtained at all positions best fits for the relative velocities $\left(v_{r}\right)$. To determine the velocity dispersion and intensities of the emission lines, we applied a leastsquares fit at each spatial location of the sum of four Gaussian curves. The four Gaussian curves were constrained to have identical widths and to have centers at the wavelengths determined by velocity fitting above, but were allowed independent amplitudes. Examples of the fits are shown as the

TABLE 2. List of observed $\mathrm{H}_{2} \mathrm{O}^{+}$lines.

\begin{tabular}{ccc}
\hline \hline$\lambda_{\text {air }} \AA$ & Band & Rotational transition \\
\hline 6198.747 & $(0,8,0)-(0,0,0)$ & $1_{10}-2_{20}$ \\
6199.388 & $(0,8,0)-(0,0,0)$ & $1_{11}-2_{21}$ \\
6200.030 & $(0,8,0)-(0,0,0)$ & $1_{10}-2_{20}$ \\
6200.543 & $(0,8,0)-(0,0,0)$ & $1_{11}-2_{21}$ \\
\hline
\end{tabular}




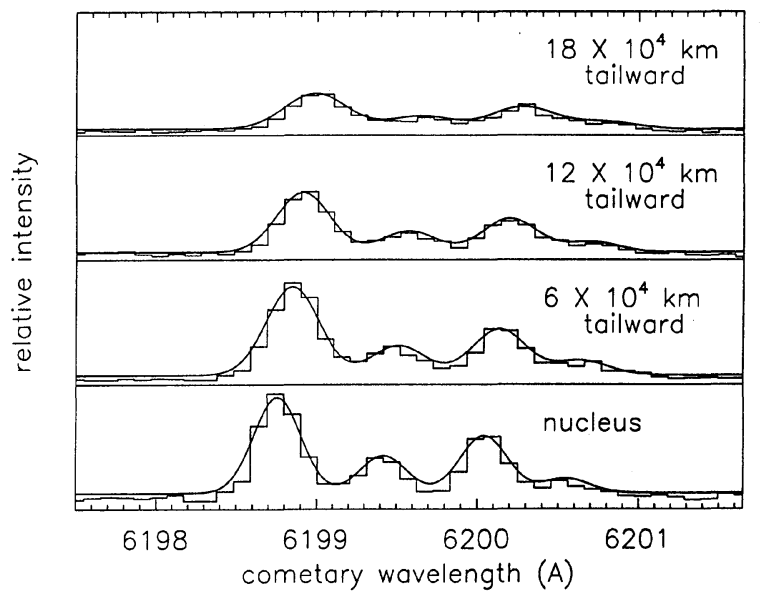

FIG. 2. One-dimensional spectra at four distances from the nucleus. The velocity shift of the spectra is clearly visible in these plots, as is the high signal to noise of these spectra. The solid lines are Gaussian fits to the spectra.

solid lines in Fig. 2. A single component Gaussian fits each of the four lines quite well, indicating an absence of any high-velocity wings to the distribution of ion velocities. For each spectrum we determined the errors in the velocity, dispersion, and intensity by using the same cross-correlation procedure on a hundred simulated two-dimensional spectra having the same velocity shifts, velocity dispersions, and line intensities, and random noise at a level determined by the measured $\mathrm{S} / \mathrm{N}$ at each location in the real spectrum. Typical errors in the central parts of the spectra are $<1 \mathrm{~km} \mathrm{~s}^{-1}$ for the relative velocities, and $\sim 1 \mathrm{~km} \mathrm{~s}^{-1}$ for the velocity dispersions. The cross-correlation method only measures relative velocities; to obtain an absolute velocity scale we checked the measured radial velocities of both cometary ions and neutral lines ([O I], $\lambda 6300$ in particular) on both long-slit and regular echelle spectra. The geocentric radial velocities agree very well with Yeoman's orbital predictions; typical differences are around $1 \mathrm{~km} \mathrm{~s}^{-1}$.

One difficulty with these data is that all emissions are line-of-sight integrations through the entire comet. To understand these projection effects we constructed simple brightness models of a comet consisting of a cylindrical tail capped by a hemispherical head, where the emission intensities fell as the square of the distance from the nucleus. Experimentation with these models and the observation geometry shows that for a purely anti-Sunward velocity, the measured velocity at the distance along the plane of the sky is extremely close to the projection of the true velocity at the distance projected onto the Sun-tail vector. In the remainder of this paper, all distances and velocities cited will be deprojected in this manner.

\section{RESULTS AND PRELIMINARY DISCUSSION OF THE $\mathrm{H}_{2} \mathrm{O}^{+}$ KINEMATICS}

\subsection{Velocities on the Sunward Side}

Figure 3 shows the measured radial velocities as a function of distance from the cometary nucleus. The dense por-

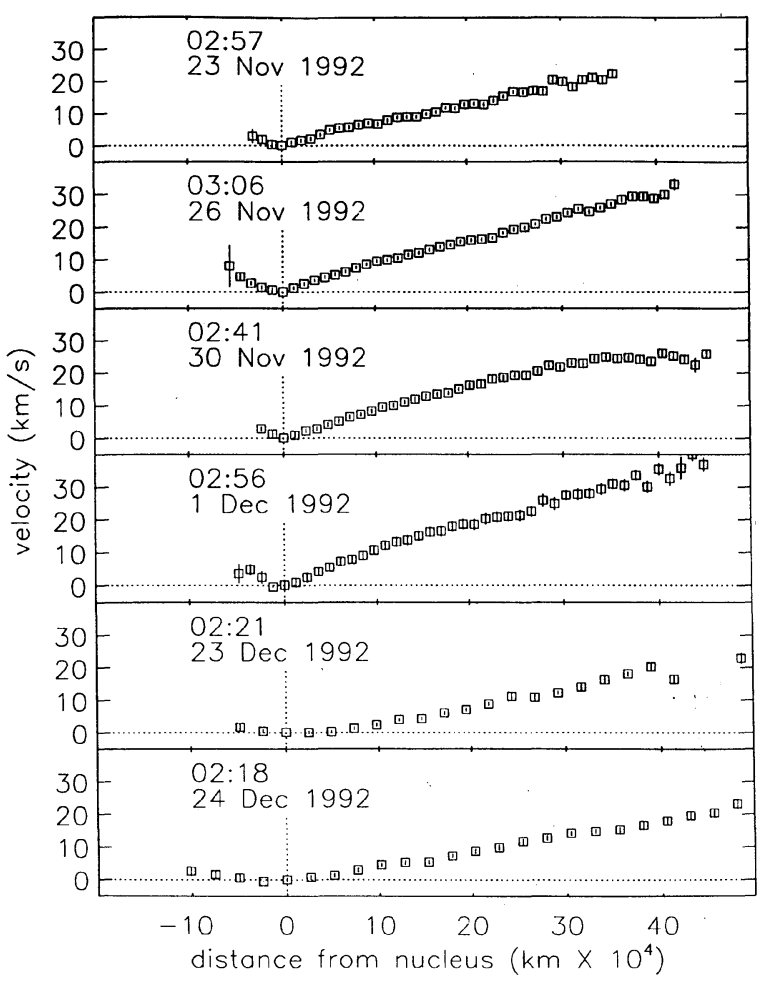

Fig. 3. Velocity shifts measured from the six best Sun-tail vector spectra. The shifts were calculated using a cross-correlation method and corrected for projection effects. The error bars shown are one sigma errors determined through a Monte Carlo method detailed in the text. The vertical dotted line denotes the position of the nucleus, and negative distances are sunward of the nucleus. The date and time (UT) of the midpoint of each observation is shown.

tions of the cometary ionosphere does not extend very far toward the Sun in typical periodic comets (McCarthy et al. 1987; DiSanti et al. 1990; Ip et al. 1985). Thus it is a small technical triumph to observe the $\mathrm{H}_{2} \mathrm{O}^{+}$velocities on the Sun side.

In typical cometary models, neutral water molecules sublime from the nucleus and flow outwards, eventually photodissociating into smaller neutral fragments or ionizing into $\mathrm{H}_{2} \mathrm{O}^{+}$. The newly created water ions are then picked up by the solar wind flow and quickly accelerated tailwards to the local solar wind velocity, determined primarily by the undisturbed solar wind speed and density, the cometary ion density, and momentum conservation.

Theoretically, three distinct boundaries exist between the region of undisturbed solar wind flow and the cometary nucleus. First is the outer interface-the bow shock-not detected by our ground-based data in any way, but well observed by the Giotto spacecraft (Flammer 1991); second, the arguably present cometopause (the radius within which mass loading of cometary ions increases rapidly and collisions with neutrals can slow the solar wind even more); and third, the ionopause or "contact surface" (perhaps $4000 \mathrm{~km}$ in radius for production rates seen in $\mathrm{P} / \mathrm{Halley}$ and $\mathrm{P} / \mathrm{Swift}-$ Tuttle). Inside the ionopause, solar wind ions and hence the solar wind magnetic fields are excluded by the pressure of ions and neutrals flowing out from the nucleus. 
All of our measurements from P/Swift-Tuttle are inside of the predicted position of the cometopause, and the smallest region-inside the ionopause-is unresolved. Nonetheless, our measurements of radial velocities on the Sunward side of the comet strikingly confirm the quick pickup of cometary ions and the deceleration of the solar wind near the nucleus; very soon after ionization the $\mathrm{H}_{2} \mathrm{O}^{+}$ions on the Sunside are already moving radially away from the Sun toward the cometary tail, in contrast to the neutral coma molecules which are generally initially seen to be expanding away from the nucleus toward the Sun (Spinrad 1991; Crovisier et al. 1993; Colom 1993; Brown \& Spinrad 1993).

\subsection{Flow on the Tailward Side}

The details of the plasma flow into cometary tails are not as well understood as those relating to the pickup and initial flow on the Sun-facing hemisphere. This is unfortunate; most of the ions end up on the tailward side. Although valuable plasma data were obtained at $8000 \mathrm{~km}$ downstream from the $\mathrm{P} /$ Giacobini-Zinner nucleus by the ICE spacecraft (von Rosenvinge et al. 1986; Meyer-Vernet et al. 1987; Smith et al. 1986), the tail side of the ionopause or the regions of the tail far from the nucleus $\left(d>10^{5} \mathrm{~km}\right)$ have not been sampled by spacecraft. Our long-slit spectra provide a first and contiguous look at the kinematics of these regions of the ion tail.

Examination of the velocities in the tailward region in comet $\mathrm{P} /$ Swift-Tuttle shows a smooth transition from low velocity increasing into the tail $\left(d>10^{5} \mathrm{~km}\right)$. On each spectrogram the tailward increase in velocity starts at the first measured point past the nucleus, thus the protected extent of any low velocity ionosphere near the nucleus must be very restricted, to $\Delta d \leqslant 10^{4} \mathrm{~km}$. The MHD models of Schmidt \& Wegmann (1991) and Wegmann et al. (1987) predict a teardrop shape to the shielded ionopause, with the tailward section extending to around $2 \times 10^{4} \mathrm{~km}$ downstream for a Halley-type comet. This undisturbed region should not allow ion pickup by the solar wind. We do have sufficient spatial resolution to observe the radial velocity of $\mathrm{H}_{2} \mathrm{O}^{+}$ions within this near tail, and they are consistently near $\Delta v=+2 \mathrm{~km} \mathrm{~s}^{-1}$ (velocity towards the tail). It is difficult, however, to conclude from this that we are seeing incomplete shielding inside the ionopause; as previously discussed, all of the measurements are integrated through a cometary line of sight. We concluded earlier that for regions of simple and wellunderstood geometry, these line-of-sight measurements were essentially equivalent to measurements along the Sun-tail axis. This region near the nucleus is neither simple nor well understood, thus understanding the differences between true effects and those that are only due to the line-of-sight measurement is impossible. In particular, we suspect that the "measurement" of a velocity flow in the tailside of the ionopause is likely a consequence of observing ion flow slipping past the ionosphere in the front and back of our line of sight.

At larger distances down the tail, the bulk speed increases, in accord with the Schmidt \& Wegmann models. The striking, zeroth-order message of the ion kinematics on the tail side, where ions spend the most time, is the steady increase

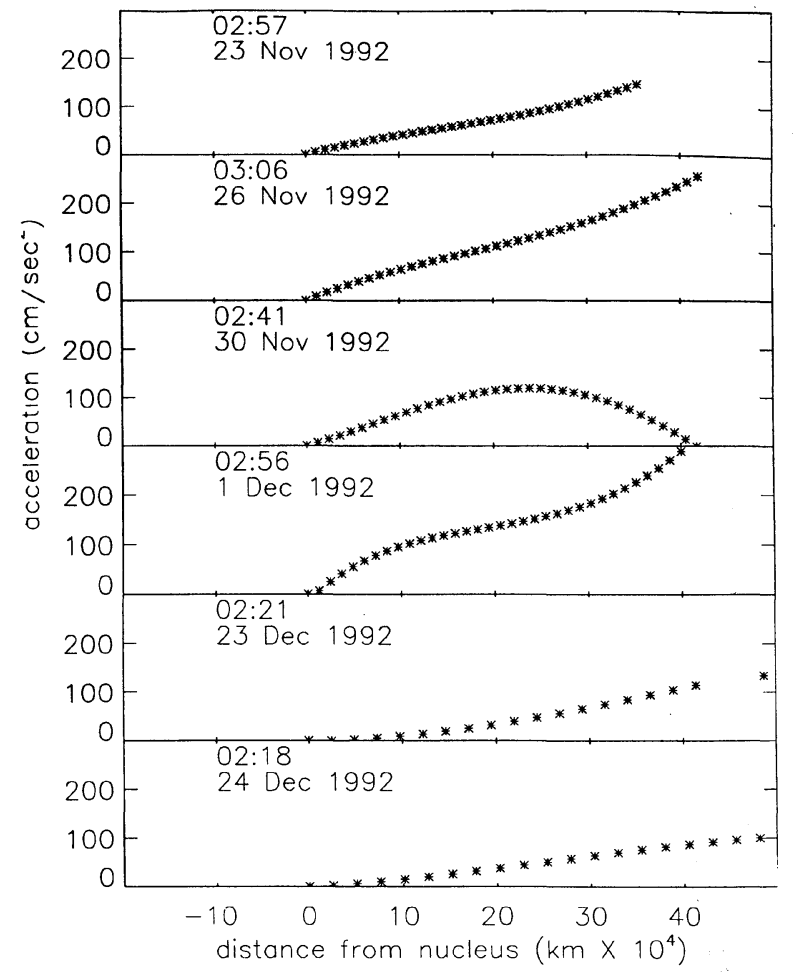

FIG. 4. Accelerations measured from the velocity shifts in the best six spectra. The accelerations were calculated by first smoothing the measured velocities and then determining the instantaneous acceleration at each point.

in $v_{r}$ down the tail on all nights of observation. There are systematic differences in the slope of $v_{r}$ versus distance, however. The general trend shown in Fig. 4 leads us to Fig 5, the ion accelerations down the tail, calculated by first smoothing the velocity data. Accelerations in cometary tails have long been surmised by proper motion studies of tail features in bright comets of the past. The proper motions, if interpretated as bulk speeds down the tail, usually led to measured accelerations from 50 to $500 \mathrm{~cm} \mathrm{~s}^{-2}$ (Brandt \& Mendis 1979; Jockers 1981; Celnick \& Schmidt-Kaler 1987).

The accelerations derived from our smoothed velocities generally indicate a systematic increase with tailward distance. The acceleration starts abruptly at the nucleus. At a distance of about $10^{5} \mathrm{~km}$, the typical acceleration approaches $40 \mathrm{~cm} \mathrm{~s}^{-2}$ (to factor of 2), while near the end of our spectra, at $d=4 \times 10^{5} \mathrm{~km}$, the accelerations are greater than 100 $\mathrm{cm} \mathrm{s}^{-2}$ generally. The accelerations clearly do not rise at the same rates on different observing nights. These data suggest occasional discontinuities in velocity/accelerationespecially noted on the 1992 December 1 spectrum at a tailward distance of $15 \times 10^{4} \mathrm{~km}$ and some evidence of the same nature can be seen between 10 and $40 \times 10^{4} \mathrm{~km}$ out on several occasions. The gradual increase in acceleration down the near tail may differ from the constant accelerations observed for disconnection events at larger $\left(>5 \times 10^{5} \mathrm{~km}\right)$ distances down the ion tail. 


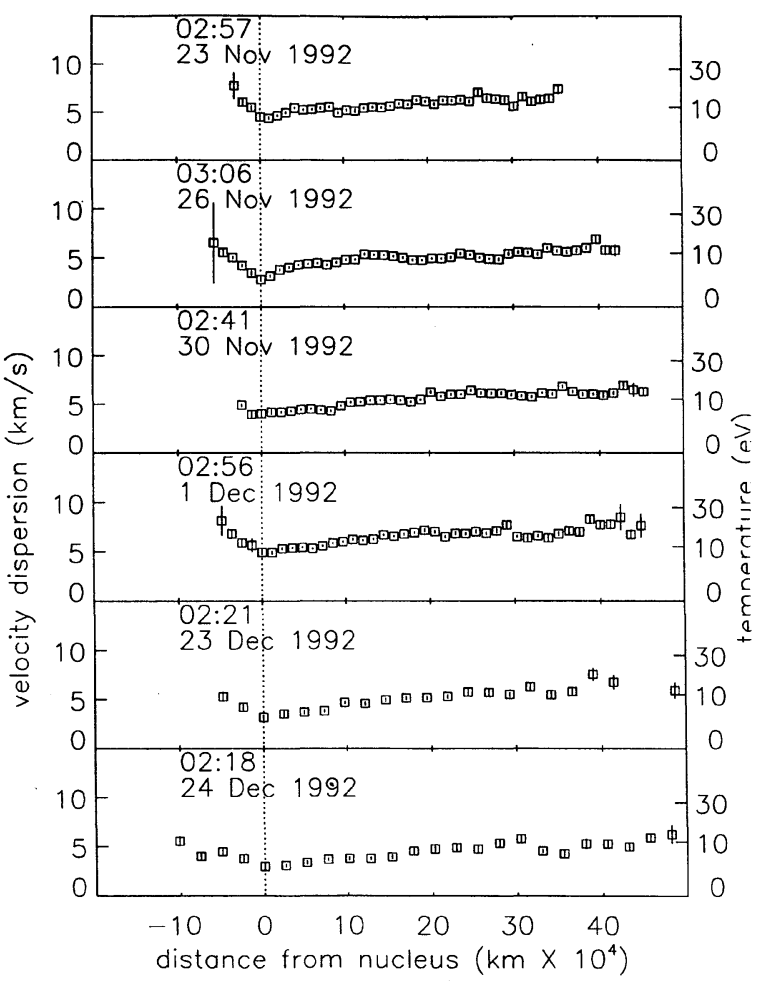

Fig. 5. The velocity dispersion determined from Gaussian fits to the $\mathrm{H}_{2} \mathrm{O}^{+}$ spectra. The dispersions have been deconvolved using a Gaussian line profile having a width of $4 \mathrm{~km} \mathrm{~s}^{-1}$ measured from a reference thorium-argon lamp. The isotropized ion temperature is shown on the right axis. The error bars shown are one sigma errors. Note the rapid heating sunward of the nucleus and the slow heating down the tail.

\subsection{Ion Temperature Measurements}

Equating the velocity dispersion, $\sigma_{r}$, with isotropized motion of the water ions in Comet $\mathrm{P} / \mathrm{Swift}-\mathrm{Tuttle}$, we can calculate ion temperatures, $T_{i}$, in the comet,

$$
k T_{i}=m_{i} \sigma^{2}
$$

Figure 5 shows these velocity dispersions, deconvolved from the instrumental profile using a Gaussian profile with a width of $4 \mathrm{~km} \mathrm{~s}^{-1}$ measured from a reference thorium-argon lamp.

The area of the comet with the lowest ion temperatures is clearly the nucleus. Once again, the nucleus is the hardest region in which to understand the observations because of likely difficulties with projection effects. Fortunately, we also have higher spectral and spatial resolution echelle spectra with the Lick $3 \mathrm{~m}$ telescope for the nucleus of Comet Austin (1989c) at the $\lambda 6199 \mathrm{H}_{2} \mathrm{O}^{+}$band. With $2^{\prime \prime}$ angular resolution $(\sim 500 \mathrm{~km}$ at that comet) on the comet we should resolve the ionopause quite well, and indeed the $\mathrm{H}_{2} \mathrm{O}^{+}$lines are slightly narrower than those measured in $\mathrm{P} / \mathrm{Swift}-\mathrm{Tuttle}$. Because this is a line-of-sight integration through regions of higher temperature and velocity, we believe this measurement to be an upper limit for the ion temperature at the nucleus. As even higher spatial resolution observations become available, we anticipate a better separation of the ionopause and surrounding regions.

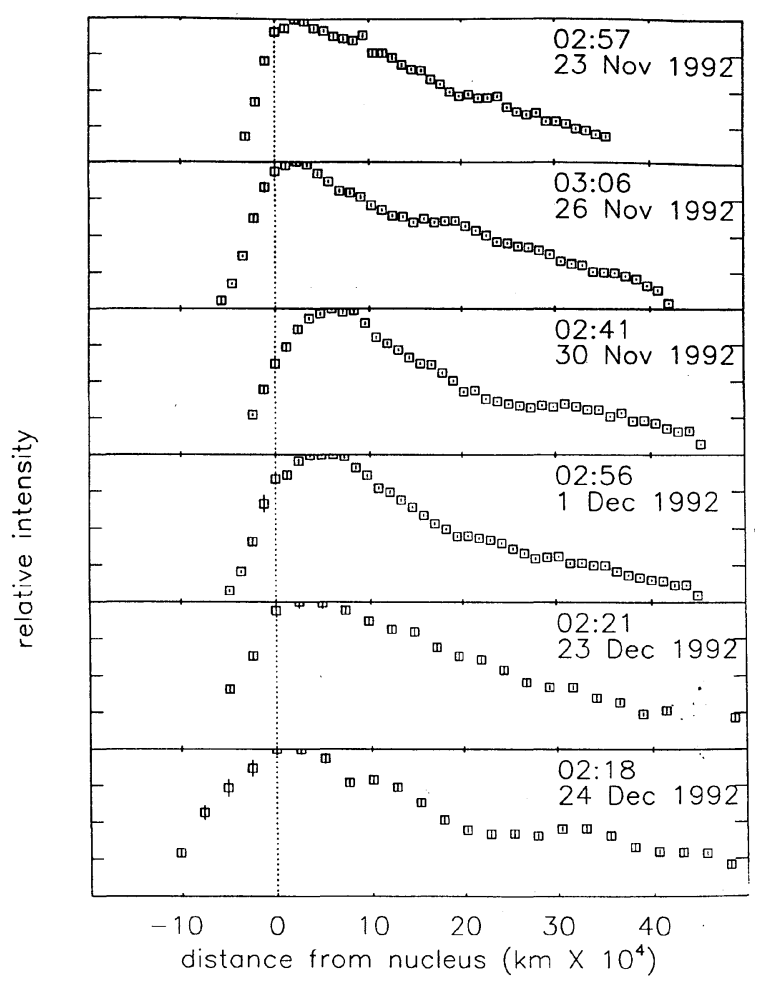

FIG. 6. The intensity of $\mathrm{H}_{2} \mathrm{O}^{+}$emission for the six best spectra. The error bars shown are one sigma random errors. Note that the peak of emission occurs tailward of the nucleus in almost all spectra.

The minimum velocity dispersion measured in Comet Austin is $\sigma_{r}=3.5 \mathrm{~km} \mathrm{~s}^{-1}$, corresponding to an upper limit minimum temperature of $T_{i}=2.7 \times 10^{4} \mathrm{~K}$, or $E_{i}=2.3 \mathrm{Ev}$.

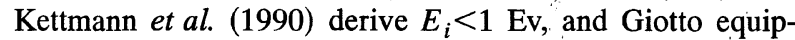
ment indicated $E_{i}=7 \mathrm{Ev}$ at a distance of $\sim 4 \times 10^{4} \mathrm{~km}$ from the Halley nucleus. Thus our upper limit observations are consistent with previous measurements. The near-nuclear MHD $T_{i}$ values in Wegmann et al. (1987) show a rapid increase from $d=3 \times 10^{3} \mathrm{~km}\left(T_{i} \sim 10 \mathrm{~K}\right)$ to $d=3 \times 10^{4} \mathrm{~km}$ $\left(T_{i}=2 \times 10^{4} \mathrm{~K}\right)$, further illustrating the difficulties in deconvolving the integrated line-of-sight measurement. The minimum ion temperature that a "smoothed" ICE spacecraft would have measured for the inner tail of Comet P/Giacobini-Zinner would have been about $2 \times 10^{4} \mathrm{~K}$.

The highest temperatures are measured on the extreme Sunside of the comet, where the ions are interacting with the still quickly flowing solar wind, and off the tail axis, where the ions are heated due to the magnetic field compression (see below). On the Sunside, we find $T_{i}=10^{5} \mathrm{~K}$, and off the tail axis (on the perpendicular spectrum), $T_{i} \simeq 3 \times 10^{5} \mathrm{~K}$. The agreement with the MHD models is good.

\subsection{Intensities}

As a by-product of our velocity measurements for the $\mathrm{H}_{2} \mathrm{O}^{+}$lines on our long-slit spectra, we also tabulated relative $\mathrm{H}_{2} \mathrm{O}^{+}$line intensities by spectrally integrating the $\mathrm{CCD}$ counts over our group of four water-ion features near $\lambda 6199$, along the slit. These intensities are shown in Fig. 6. The 
relative $\mathrm{H}_{2} \mathrm{O}^{+}$intensity profiles resemble those shown by DiSanti et al. (1990), McCarthy et al. (1987), and Spinrad \& Strauss (1986). These authors usually found the maximum ion density at or slightly tailward of the various comet nuclei; on our $\mathrm{P} /$ Swift-Tuttle spectra we find the $\mathrm{H}_{2} \mathrm{O}^{+}$intensity maximum between 1 and $4 \times 10^{4} \mathrm{~km}$ tailward. This intensity maximum is particularly well determined on these spectra because their high resolution allows us to very easily subtract out the contribution from the dust continuum. After the intensity maximum, the tailside shows a fairly slow decline (out to the limits of observation).

It is unlikely that many $\mathrm{H}_{2} \mathrm{O}^{+}$molecules once picked up by the solar wind will be lost to our view. The typical recombination length is $\approx 10^{7} \mathrm{~km}$. Some early ions could be lost near the nucleus by electron recombination in higher density regions or further ionization or recombination. So the gradual ionic intensity decrease down the tail may simply be the effect of increasing tailward speed and the shorter dwell time for $\mathrm{H}_{2} \mathrm{O}^{+}$molecules in each spatial pixel. DiSanti et al. (1990) used this hypothesis to estimate the plasma flow rate from measurements of ion intensity in the tail.

\subsection{Perpendicular Spectrum}

On December 19 and 22 1992, we obtained two good spectra with the slit centered on a region about 2 arcmin, or about $3 \times 10^{5} \mathrm{~km}$, down the tail and perpendicular to the Sun-tail axis. Figure 7 shows the velocities, ion temperatures, and intensities for the best of the two perpendicular spectra. These spectra are similar to the trajectory of the ICE spacecraft through the tail of Comet Giacobini-Zinner, though much further down the tail, and the general structure seen in the velocities, temperatures and intensities closely resemble the measurements made by Giotto. In particular, the flow in the inner portion of the tail is slow and cold while the outer portion is fast and hot. Note the substantial shear visible in the spectra.

\section{KINEMATIC CORRELATIONS}

Examination of the velocity and velocity dispersion plots indicate the day to day changes in kinematics down the tail, but also accentuate the correlation between daily $v_{r}$ and $\sigma_{r}$. The bulk flow and the isotropized random motions vary together.

Other clear-cut correlations in this kinematic study are the continuity and systematic increase in the bulk ionic flow down the tail and the increase in $\sigma_{v}$ toward all lower-density regions of the cometary ion distribution-the tail off-axis location and near the cometopause on the Sunside. These presumed pickup regions of relatively low cometary $\mathrm{H}_{2} \mathrm{O}^{+}$ density seem to be the most turbulent areas, and even their ratio of $\sigma_{r} / v_{r}$ is greatest there, with ratio values ranging up to 2-3 sunside, $\sim 1$ off the tail axis, while in the tail at $\sim 10^{5}$ $\mathrm{km}$ downstream, $\sigma / v$ is $\sim 0.7$, decreasing to $\sim 0.3$ at $3 \times 10^{5}$ $\mathrm{km}$ downstream. These kinematic ratios, while not explicitly calculated in the MHD analysis of Wegmann et al. (1987), can be visually recognized in their Fig. 3 panels of ion temperature and velocity. The qualitative spatial variation agree-

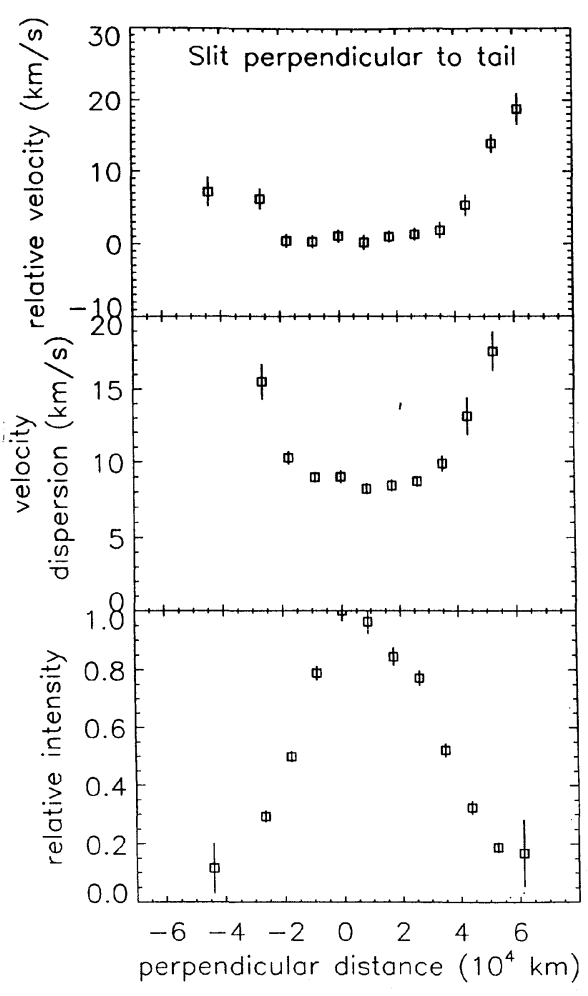

FIG. 7. The velocity, velocity dispersion, and intensity of $\mathrm{H}_{2} \mathrm{O}^{+}$emission for a spectra taken with the slit perpendicular to the Sun-tail vector and centered $3 \times 10^{5} \mathrm{~km}$ down the tail. The lowest velocity point is set to $15 \mathrm{~km} \mathrm{~s}^{-1}$ based on the data in Fig. 3. Note that the velocity dispersion measured off the tail axis is the highest measured anywhere in the comet.

ment of these two parameters strengthens our confidence in the basic physical mechanisms in these models.

The product of the intensity, $I$, and the square of the velocity dispersion is roughly constant, suggesting some sort of rough conservation of static ion pressure $\left(N_{i} k T_{i} \approx I \sigma_{r}^{2} \approx\right.$ constant $)$. Indeed, plots of the $I \sigma^{2}$ product over the comet head and near tail show fairly good constancy; certainly this product is better behaved than either independent variable.

There are also considerable systematic changes in the tailward bulk flow over periods of weeks; our spectra on 1992 UT dates November 23, 26, and 30 and December 1 show a fast tailward flow, parametrized by a mean velocity at $d=10^{5}$ $\mathrm{km}$ tailward of the nucleus $v_{5}=9 \mathrm{~km} \mathrm{~s}^{-1}$, while the spectra of December 23 and 24 have $v_{5}=3.5 \mathrm{~km} \mathrm{~s}^{-1}$. The accelerations and $\sigma_{r}$ are also bimodal when grouped this way, with the acceleration at $d=10^{5} \mathrm{~km}, a_{5} \approx 70 \mathrm{~cm} \mathrm{~s}^{-2}$ in the fast group and $a_{5} \approx 10 \mathrm{~cm} \mathrm{~s}^{-2}$ in the slow pair of observations.

To compare these velocities with absolute intensities, we have calculated a set of absolute ion brightnesses, comparing the four $\mathrm{H}_{2} \mathrm{O}^{+}$lines to the local continuum produced by the nucleus dust of Comet P/Swift-Tuttle. The implicit assumption is that dust production, integrated over a moderate time interval, is constant. Limited experience indicates that the ionic stream changes more radically than the dust production at $r \simeq 1 \mathrm{AU}$. We present the results in Fig. 8 . Note the marked anticorrelation of the "absolute" intensity parameter, $I$, with 


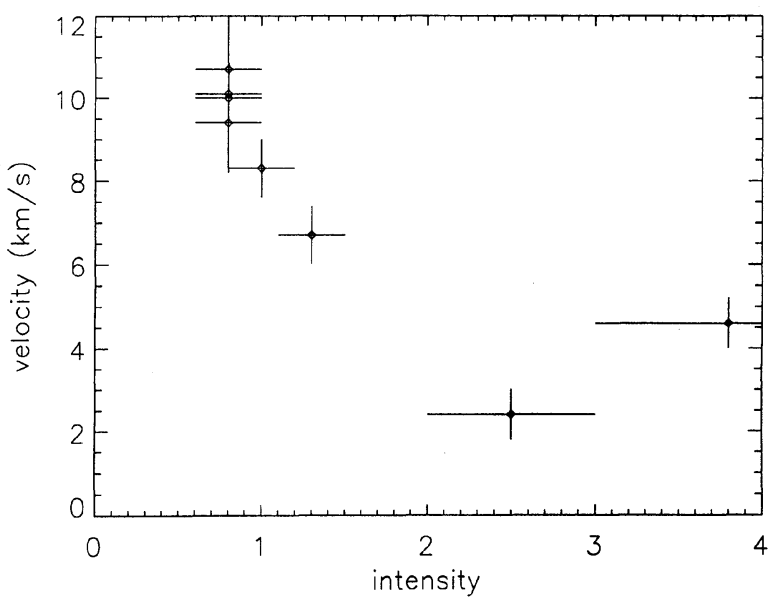

FIG. 8. A comparison of the peak intensity of $\mathrm{H}_{2} \mathrm{O}^{+}$emission and the tailward velocity at a distance of $10^{5} \mathrm{~km}$ tailward. The anticorrelation between velocity and intensity suggests that variable mass loading of cometary ions is responsible for variations in the flow velocity.

the flow velocity at $d=10^{5} \mathrm{~km}$ down the tail.

This bulk flow anticorrelation with $I$ is slightly stronger than the $\sigma$ or $\sigma / V$ ratio anticorrelation with intensity. We can imagine two possible interpretations of these systematic trends. The most likely scenario is that we are actually witnessing the direct effect of the mass loading of cometary ions into the solar wind, with the deceleration of the latter being greater on occasions where the cometary ion production/ density has increased. Alternatively, we could simply be viewing the variability in the undisturbed solar wind velocity; it may have just been blowing harder in late November than late December. Unfortunately, the comet was not in the ecliptic plane during our observing sessions $\left(i=113^{\circ}\right)$, so no direct solar wind velocities from extant spacecraft are available and relevant.

One small datum in favor of the mass-loading change scenario is that the neutral gas and dust production rates inferred from the visual light curve of $\mathrm{P} / \mathrm{Swift}-\mathrm{Tuttle}$ (DiCicco 1993; Sekanina 1993) suggest a slight increase in the production rates by 1992 late December (post perihelion by +22 days). Whether this modest $Q$ difference actually lead to an increase in $\mathrm{H}_{2} \mathrm{O}^{+}$ion densities by December 23 is uncertain.

\section{PHYSICAL DEDUCTIONS AND INFERENCES ABOUT THE AMPLIFIED $B$ FIELD}

We have attempted to infer some semiquantitative values for the enhanced interplanetary magnetic field (IMF) carried over the cometary barrier and compressed past there by assuming two sorts of equipartition situations and using our measured ionic temperatures to calculate the ionic pressures and the compressed $B$ field.

\subsection{Sunside Field}

First we examine the ram-pressure situation on the daylight hemisphere of $\mathrm{P} / \mathrm{Swift}-\mathrm{Tuttle}$. We equate the solar wind
TABLE 3. Transverse $B_{\text {static }}$ field computations.

\begin{tabular}{lcccc}
\hline \hline Comet & $\begin{array}{c}\text { Spatial } \\
\text { position }\end{array}$ & $\begin{array}{c}B_{L} \text { (assumed) } \\
(\mathrm{nT})\end{array}$ & $\begin{array}{c}\sigma_{\text {ion }} \\
\left(\mathrm{km} \mathrm{s}^{-1}\right)\end{array}$ & $\begin{array}{c}B_{\text {static }} \\
(\mathrm{nT})\end{array}$ \\
\hline Austin & nucleus & 80 & 4 & 79 \\
$(1990 \mathrm{~V})$ & nucleus & 60 & 4 & 57 \\
P/Swift-Tuttle & on tail axis & 80 & 8 & 73 \\
& off tail axis & 80 & 14 & 70 \\
P/Swift-Tuttle & on tail axis & 60 & 8 & 50 \\
& off tail axis & 60 & 14 & 45 \\
\hline
\end{tabular}

ram pressure to the sum of the cometary static ion pressure and magnetic pressure near the cometopause on the daylight hemisphere. In pressure balance,

$$
\rho_{\mathrm{sw}} v_{\mathrm{sw}}^{2}=n_{i} k T_{i}+B^{2} / 8 \pi,
$$

where $\rho_{\mathrm{sw}}$ and $v_{\mathrm{sw}}$ are the solar wind density and velocity, $n_{i}$ and $T_{i}$ are the ion number density and temperature, and $B$ is the compressed magnetic field strength. The $\mathrm{H}_{2} \mathrm{O}^{+}$ion density there is fairly uncertain; we use a value of $n_{i}=30 \mathrm{~cm}^{-3}$. For the solar wind parameters, we use average values of $v_{\mathrm{sw}}=400 \mathrm{~km} \mathrm{~s}^{-1}$ and $\rho_{\mathrm{sw}}=10^{-23} \mathrm{~g} \mathrm{~cm}^{-3}$. The ion temperature is our maximum measured value of $8.3 \times 10^{4} \mathrm{~K}$. Using these parameters, we find that the magnetic field required is of the order of $50 \mathrm{nT}$, and that the magnetic pressure dominates that due to the static ions field. Because of the simplistic nature of this calculation and the uncertainties in the parameters, this estimate is probably uncertain by factors of several. Nonetheless, the deduced field is within an order of magnitude of that measured by Giotto along its sunward side track through the Halley cometopause.

\subsection{Tailside Transverse Field}

We can also estimate the compressed $B$ field in the cometary tail and near the nucleus, following an approximation derived by Siscoe et al. (1986), who use a static pressure balance equation under several assumptions. They ignore the possible contribution by free electrons to the pressure (since $T_{i}>T_{e}$ in the extant MHD models this assumption is probably justifiable). More importantly, Siscoe et al. assume a ficticious transverse compressed solar wind field, $B_{L}$, present conceptually in the "comet as an obstruction" model, in the absence of ion plasma pressure. Then $B_{L}$ is the "lobe field," and

$$
n_{i} k T_{i}+B_{\text {static }}^{2} / 8 \pi=B_{L}^{2} / 8 \pi,
$$

where $B_{\text {static }}$ is the static pressure balancing field to be determined. We use our $\sigma_{r}$ 's to determine $T_{i}$, and we assume $n_{i}(\max )=200 \mathrm{~cm}^{-3}$ for $\mathrm{P} / \mathrm{Swift}-\mathrm{Tuttle}$, in coarse analogy to Comet P/Giacobini-Zinner. With the same arbitrariness as in Siscoe et al. (1986) we assume, sequentially, cases with $B_{L}=80$ and $60 \mathrm{nT}$ (factors of $\approx 10$ in $B$-field compression of the original IMF). On the tail axis, we find typical values of $B_{\text {static }}=60 \mathrm{nT}$, while off the tail axis, using an estimate of $n_{i}=100 \mathrm{~cm}^{-3}$, we find $B_{\text {static }}=50 \mathrm{nT}$.

We can, of course, parametrize the assumed $B_{L}$ over a range that allows $B_{\text {static }}$ offaxis to remain finite. In Table 3 we use this approximation to estimate the static transverse fields 
at various locations in two comets with dispersion data. The derived static fields in Table 3 are quite consistent with those derived without $\sigma_{r}$ measures but using explicitly measured ion densities (from the ICE spacecraft data) by Siscoe $e t$ al.

We have already noted the constancy of the ion pressure component over the P/Swift-Tuttle ionosphere (Sunside and tail). The $B$-field pressure does vary more, but is of a similar order of magnitude.

\section{INCREASES IN THE TAIL DIRECTED ION ACCELERATIONS}

Accelerations along cometary tails have frequently been derived from proper motion measures of ion knots (Brandt \& Mendis 1979; Farnham \& Meech 1994). A few spectroscopic velocity comparisons have also yielded accelerations, indeed rather small ones, for P/Halley in 1985 and 1986 (Wyckoff \& Lindholm 1994; Scherb et al. 1990).

A glance at Fig. 5 shows the general increase in ion accelerations down the comet tail. These data show a systematic increase in acceleration from some $80 \mathrm{~cm} \mathrm{~s}^{-2}$ at $2 \times 10^{5}$ $\mathrm{km}$ distance to $>120 \mathrm{~cm} \mathrm{~s}^{-2}$ at $d>4 \times 10^{5} \mathrm{~km}$ downstream. There are considerable changes from night to night. What is the cause of these changes in the ion acceleration?

Cravens (1991) and Jockers (1982) suggest the $J \times B$ curvature force, with draping/converging field lines driving the ions enmeshed in the solar wind. Of course other global forces could in principal be important; Wyckoff \& Lindholm (1994) have ruled out radiation pressure on $\mathrm{H}_{2} \mathrm{O}^{+}$accounting for even $1 \%$ of our measured accelerations. Another possibility (Ip \& Axford 1990) would be shock accelerating which under favorable circumstances, could work like a Fermi accelerator, bouncing the changed particles like tennis balls rebounding from moving reflecting walls. Tsurutani et al. (1989) and Tsurutani (1991) have suggested plasma waves and instabilities (on less-global cometary scales) as likely acceleration mechanisms to increase tailward ion accelerations. The MHD models (Schmidt-Vogt 1989) seem to obtain tailward accelerations simply through hydrodynamic and magnetic pressures, though the specific mechanism is difficult to discern.

We have one indirect clue to offer from our kinematic measures along the tail. That is the bulk flow speed increases more rapidly down the tail than does the turbulence of the smaller-scale gyrational motion. Thus we would favor a more global mechanism, like the gradual field line convergence, suggested early by Wurm and others from the geometric appearance of many cometary tail rays.

Finally, we may update an order-of-magnitude calculation initially suggested by Iaffe (1968); using an implicit model of the convergent $B$-field gradient, one can calculate the acceleration induced for water group ions over specific linear scales in the comet tail. Using the mean static $B$ field of Sec. $4(50 \mathrm{nT})$, and with an ion density $n_{i}=100 \mathrm{~cm}^{-3}$ at $d=3 \times 10^{5} \mathrm{~km}$, we employ

$$
a=B^{2} / 2 \pi n_{i} m_{i} d,
$$

where $m_{i}$ is the mass of the water ion, with our values to calculate $a \approx 400 \mathrm{~cm} \mathrm{~s}^{-2}$ which is within a factor of 2 of the

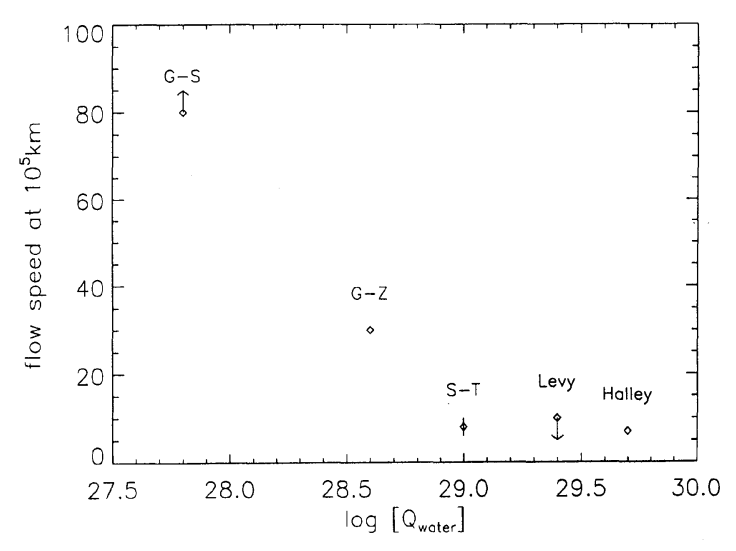

FIG. 9. A comparison of the production rates (in water molecules per second) of five comets with the flow speed at tailside distances $<10^{5} \mathrm{~km}$ from the nucleus. For comet Grigg-Skjellerup (GS), the velocity was measured by Giotto at a distance of about $200-500 \mathrm{~km}$, so the measured velocity is a strong lower limit to the velocity at $10^{5} \mathrm{~km}$ (Johnstone et al. 1993). The velocity for Comet Giacobini-Zinner (GZ) was measured by the ICE probe (Bame et al. 1986). The Swift-Tuttle (ST) measurements are from this paper, the Levy upper limit is a measurement at $10^{5} \mathrm{~km}$ from Rauer \& Jockers (1993), and the Halley measurement is from Wyckoff \& Lindholm (1994).

acceleration observed at $d=3 \times 10^{5} \mathrm{~km}$ on the nights of highest radial velocity (cf. Figs. 4 and 5 ).

\section{SOME COMPARISONS WITH ION TAIL SPEEDS IN OTHER COMETS}

Besides the spacecraft single trajectory measures of ion bulk flow and $T_{i}$ in Comets $\mathrm{P} /$ Halley, P/Giacobini-Zinner, and $\mathrm{P} /$ Grigg-Skjellerup, several observers have obtained broad-beam Fabry-Perot spectra and some short-slit high resolution spectra from the ground for fairly bright comets. In Fig. 9 we plot bulk flow speeds measured in the $\mathrm{H}_{2} \mathrm{O}^{+}$tail, or the ICE/Giotto 2 midtail cometocentral speed measured by the spacecraft against the cometary production rate for water, $Q_{\text {water }}$, for each. The plotted velocities are for a distance of approximately $10^{5} \mathrm{~km}$ down the tail, except for the velocity of Comet Grigg-Skjellerup, which was measured much closer to the nucleus and therefore provides a strong lower limit to the velocity at $10^{5} \mathrm{~km}$. There is some correlation in the sense anticipated; the comets with the highest production rates might also have the lowest tailward velocities, but because of the difficulties in comparing widely varying types of observations, we are incapable of determining this with certainty. Further ground-based radial velocities, similar to those we report for P/Swift-Tuttle, are needed.

\section{SUMMARY}

In this paper we have presented observations of the ion tail and Comet $\mathrm{P} /$ Swift-Tuttle and several results from interpretation of the observations. We list here a summary of our conclusions.

(1) The measured velocities show that the accelerations observed in comet tails are indeed true bulk accelerations and not just wave phenomena. 
(2) On most nights, the tailward acceleration increases almost linearly with distance down the tail.

(3) The quick decceleration of the solar wind and heating of cometary ions Sunward of the nucleus is observed from the ground for the first time.

(4) Densities of $\mathrm{H}_{2} \mathrm{O}^{+}$peak tailward of the nucleus on all nights of observation.

(5) Ion temperatures in the comet increase with distance from the nucleus and with distance from the Sun-tail axis.

(6) From the ion temperature measurements we can estimate values for the magnetic field which are in rough accord with spacecraft observations and detailed MHD models.

(7) The ion flow speed seems to be anticorrelated with the ion intensity and possibly with the water production rate, suggesting that the mass loading rate controls the flow speed.

\section{FUTURE IMPROVEMENTS IN COMETARY PLASMA OBSERVATIONS}

We regret the lack of simultaneous narrow-band $\mathrm{H}_{2} \mathrm{O}^{+}$ images of $\mathrm{P} / \mathrm{Swift}-\mathrm{Tuttle}$. Had we had images, a detailed analysis of the kinks and wiggles in the spectra would have been attempted, perhaps associating them with tail condensations or other features. We hope in the future to obtain such simultaneous imaging and spectroscopy, but in practice any of these types of observations of bright comets is difficult because the usual very short time between comet discovery and observation often prevents adequate preparation.
Imaging, along with good $\mathrm{S} / \mathrm{N}$ spectra on perpendicular cuts down the cometary tail, could be used to try to differentiate any kinematic differences between the occasionally strong tail rays and the general, more diffuse, tail plasma. Are their $\mathrm{H}_{2} \mathrm{O}^{+}$accelerations different?

Higher spatial resolution observations would allow us to distinguish interesting regions very close to the nucleus. For example, the variations in $\sigma, V$ at the contact surface could be resolved if we use an echelle spectrograph with a smaller pixel size on a nearby bright comet. This is technically feasible for naked-eye comets observed with 4-10 m class telescopes. The rapid decrease in ion density on the Sunside might also be amenable to similar observational strategy, perhaps allowing us to observe the cometopause boundary.

Finally, it would be valuable to trace the temperature and velocity structure of a heavier ion, like $\mathrm{CO}^{+}$or even $\mathrm{CO}_{2}^{+}$, at the same spatial positions as the water ions. This will be a moderately difficult task, but again would be possible for a very bright comet.

We wish to thank Donald Yeomans for providing a very accurate ephemeris, Tony Misch for his continued assistance with cometary observations, and Klaus Jockers for suggesting a search for mass loading effects. Valuable discussions with Marcia Neugebauer, Bruce Tsurutani, and Sue Wyckoff are graciously acknowledged. H.S. and M.E.B. are supported by NASA Grant No. NAGW-2883.

\section{REFERENCES}

Bame, S. J., Anderson, R. C., Asbridge, J. R., Baker, D. N., \& Feldman, W. C. 1986, Science, 232, 356

Brandt, J., \& Mendis, D. A. 1979, in Solar System Physics, Vol. II, edited by C. F. Kennel, L. J. Lanzerotti, and E. N. Parker (North-Holland, Amsterdam), p. 254

Brown, M. E., Johns, C. M., \& Spinrad, H. 1993, Geophys. Res. Lett., 20, 1003

Brown, M. E., \& Spinrad, H. 1993, Icarus, 104, 197

Celnick, W. E., \& Schmidt-Kaler, T. 1987, A\&A, 187, 233

Colom, P. 1993, IAU Circular, No. 5664

Cravens, T. E. 1991, in Comets in the Post-Halley Era, edited by R. L. Newburn, et al. (Kluwer, Dordrecht), p. 1211

Crovisier, J., et al. 1993, BAAS, 25, 1048

DiCicco, D. 1993, S \& T, July 1993, 107

DiSanti, M. A., Fink, U., \& Schultz, A. B. 1990, Icarus, 86, 152

Farnham, T. L., \& Meech, K. J. 1994, ApJ (in press)

Flammer, K. R. 1991, in Comets in the Post-Halley Era, edited by R. L. Newburn, et al. (Kluwer, Dordrecht), p. 1125

Harwit, M., \& Hoyle, F. 1962, ApJ, 135, 875

Herbig, G. H. 1990, AJ, 99, 1262

Iaffe, Z. M. 1968, SVA, 11, 1044

Ip, W.-H., \& Axford, W. I. 1990, in Physics and Chemistry of Comets, edited by W. F. Huebner, (Springer, Berlin), p. 177

Ip, W.-H., Fink, U., \& Johnson, J. R. 1985, ApJ, 293, 609

Jockers, K. 1982, Icarus, 47, 397

Johnstone, A. D., Coates, A. J., Ehuddleston, D., Jockers, K., \& Wilken, B. 1993, A\&A, 273, L1

Kettman, G., et al. 1990, Annales Geophysical, 8, 229

McCarthy, P., Strauss, M. A., \& Spinrad, H. 1987, in ESLAB Symposium of the Exploration of Halley's Comet, ESASP-200, 87
Meyer-Vernet, N., Strauss, M. A., Steinberg, J. L., Spinrad, H., \& McCarthy, P. J. 1987, AJ, 92,474

Rauer, H., \& Jockers, K. 1993, Icarus, 102, 117

Scherb, F., Magee-Sauer, K., Roesler, F. L., \& Harlander, J. 1990, Icarus, 86, 172

Schmidt, H. U., \& Wegmann, R. 1991, in Cometary Plasma Processes, edited by A. D. Johnstone (AGU Publications, Washington), p. 49

Schmidt-Vogt, M. 1989, A\&A 210, 433

Sekanina, Z. 1993, private communication

Siscoe, G. L., Slavin, J. A., Smith, E. J., Tsurutani, B. T., Jones, D. E., \& Mendis, D. A. 1986, Geophys. Res. Lett., 13, 287

Smith, E. J., Tsurutani, B. T., Slavin, J. A., Jones, D. E., Siscoe, G. L., \& Mendis, D. A. 1986, Science, 232, 382

Spinrad, H. 1991, AJ, 102, 1207

Spinrad, H., \& Strauss, M. A. 1986, in Asteroids, Comets, Meteors II, edited by C.-I. Lagerkvist, B. A. Lindblad, H. Lundstedt, and H. Rickman, (Uppsala Universitat, Upsala), p. 443

Tsurutani, B. 1991, in Comets in the Post-Halley Era, edited by R. L. Newburn, et al. (Kluwer, Dordrecht), p. 1171

Tsurutani, B. T., Page, D. E., Smit, E. J., Goldstein, B. E., \& Brillca, A. C. 1989, J. Geophys. Res., 94, 18

Vogt, S. 1987, PASP, 99, 1214

von Rosenvinge, T. T., Brandt, J. C., \& Farquhar, R. W. 1986, Science, 232, 353

Wegmann, R., Schmidt, H. U., Huebner, W. F., \& Boice, D. C. 1987, A\&A, 187,339

Wyckoff, S., \& Lindholm, E. 1994, in preparation 